\title{
Knuckle technique guided by intravascular ultrasound for in-stent restenosis occlusion treatment
}

\author{
Mladen Tasic, Miodrag J. Sreckovic, Nikola Jagic, Vladimir Miloradovic, Dusan Nikolic \\ Clinical Centre Kragujevac, Kragujevac, Serbia
}

Postep Kardiol Inter 2015; 11, 1 (39): 58-61 DOI: $10.5114 /$ pwki.2015.49188

\begin{abstract}
A bstract
One of the rarest lesions is in-stent restenosis chronic total occlusion (СТO). Limited data suggest that the treatment success rate is dependent on the possibility to cross into the lumen of an occluded stent, and the decision about what technique to use varies by operator preference. The knuckle technique is used to create a deliberate dissection plane in various CTO techniques. A guide wire is pushed until a complex loop is formed and advanced through the lesion. In this report we present a case where a knuckle wire guided by intravascular ultrasound control is used to penetrate the distal cap in an in-stent restenosis CTO lesion.
\end{abstract}

Key words: in-stent restenosis, chronic total occlusion, knuckle technique, intravascular ultrasound.

\section{Introduction}

Chronic total occlusion (CTO) is considered one of the most difficult lesion types to treat, with success rates varying between $60 \%$ and $90 \%$. Procedure success depends on operator expertise, increasing with operator's experience [1]. In-stent restenosis (ISR) occurs at rate of fewer than $10 \%$ in drug-eluting stents (DES) and is primarily related to focal neointimal proliferation. This is somewhat in contrast to the diffuse restenosis (ISR) pattern in bare metal stents (BMS) resulting in fewer clinically significant target vessel revascularisations. Although a focal pattern is observed in most cases primarily to the distal edges of DES, diffuse proliferation or total occlusion is still present in $20 \%$ of cases of ISR [2]. While different in localisation, restentotic plaque is similar in both stent types, consisting mostly of smooth muscle cells and fibrolipidic areas rich in collagen and reticular fibres [3]. One of the rarest CTO lesion is in-stent restenosis chronic total occlusion - ISR CTO (1.7\% of all lesions and around $5-10 \%$ of all CTOs) [4]. Limited data suggest that the treatment success rate is dependent on the possibility to cross into the lumen of the occluded stent, and the decision about the technique to use varies by operator preference.

The knuckle technique is used to create a deliberate dissection plane in various CTO techniques, and the wire, usu- ally a polymer-jacketed guide wire, is pushed until a complex loop is formed and advanced through the lesion [5].

We present a case where a knuckle wire is used to penetrate the distal cap in an ISR CTO lesion.

\section{Case report}

A 69-year-old woman presented to the cath lab due to exertion chest pain. A year previously the patient had successful percutaneous coronary intervention $(\mathrm{PCl})$ of a large obtuse marginal (OM) branch of the left circumflex artery (LCX) in the setting of acute myocardial infarction (Figure 1). Echocardiography revealed a hypokinetic lateral wall with slightly reduced ejection fraction. Coronary angiography showed a large occluded OM branch with visible stent struts. The occlusion extended throughout the stent with slow antegrade flow showing distal segment of artery (Figure 2). The treatment procedure was started using a femoral approach with a $6 \mathrm{Fr}$ EBU guiding catheter and a Fielder XT guidewire with the support of a Finecross microcatheter (Terumo interventional systems). Due to flush occlusion of the ostial OM branch, it was extremely difficult to negotiate and penetrate the proximal cap from the LCX main branch 2-3 mm into the occlusion. The Fielder XT guidewire was exchanged for ASAHI MiracleBros 3 and ASAHI Confian-

\section{Corresponding author:}

Miodrag J. Sreckovic MD, Clinical Centre Kragujevac, Kumanovska 5/4, 34000 Kragujevac, Serbia, phone: 381693371777 ,

e-mail: sreckovic7@gmail.com

Received: 18.06.2014, accepted: 18.07.2014. 


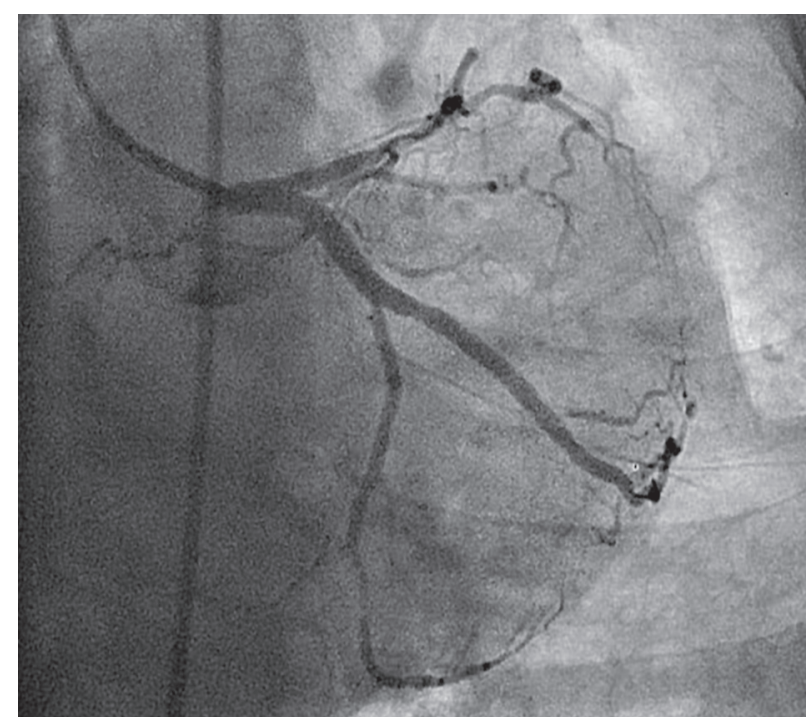

Figure 1. $\mathrm{pPCl} \mathrm{OM}$ was done a year ago

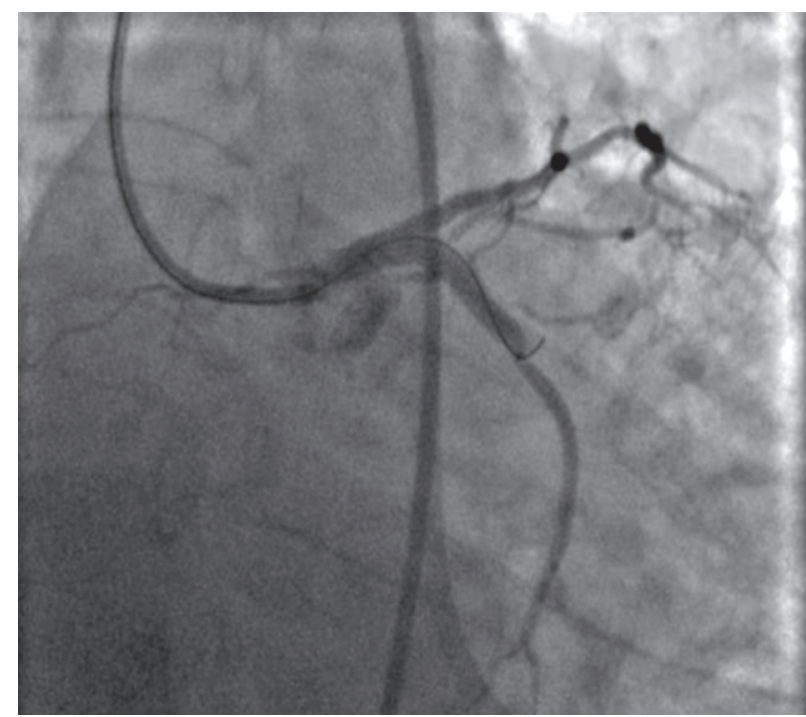

Figure 3. Failure to cross CTO

za PRO 12 guidewires, but both failed (Figure 3). Using stent struts as markers of the vessel border we managed to push the wire further distally, but we were unable to get it past the very hard distal cap. After verification of the position of the wire tip, a microcatheter was pushed into the stented segment and the Fielder XT wire was reintroduced (Figure 4). Movement of the microcatheter made enough space for us to bend the wire tip and form a triple knuckle, and then to carefully push through the distal cap and into the distal segment. After successfully passing the microcatheter and checking the intraluminal position of the system by aspiration of the blood, we exchanged the wire for a standard Runthrough guidewire and pre-dilated the whole segment (Figure 5). For better understanding of the vessel anatomy and achieved

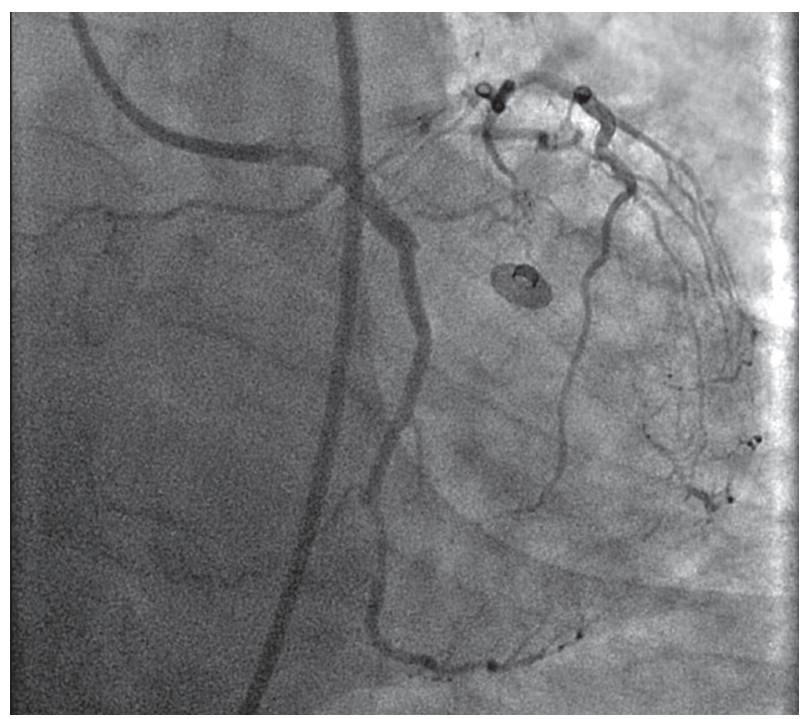

Figure 2. Occluded OM branch, visible stent struts

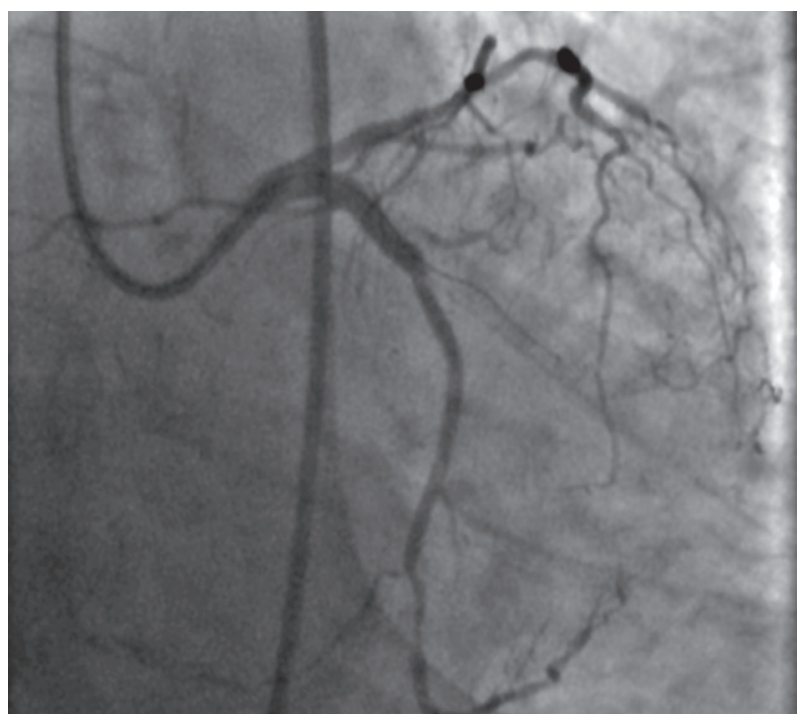

Figure 4. Passing of the wire after knuckling

results, an intravascular ultrasound (IVUS) examination was performed (Figure 6), revealing that the guidewire was properly positioned in the stent. Intravascular ultrasound examination also showed the most distal stent strut with large neointima (Figure 7), the mid section of the treated segment with unopposed stent strut as a target for restenosis, stent thrombosis with a kind of flap as a consequence of the knuckle, and the more proximal part showing mixed plaque characteristics (Figure 8). Finally, DES was implanted in the OM branch using the stopper balloon in LCx for ostial positioning, and we also treated distal LCX (Figure 9). The patient was discharged the next day with no electrocardiographic changes or enzyme elevation. We also performed 1- and 3-month follow-up, and the patient remains symptom free. 


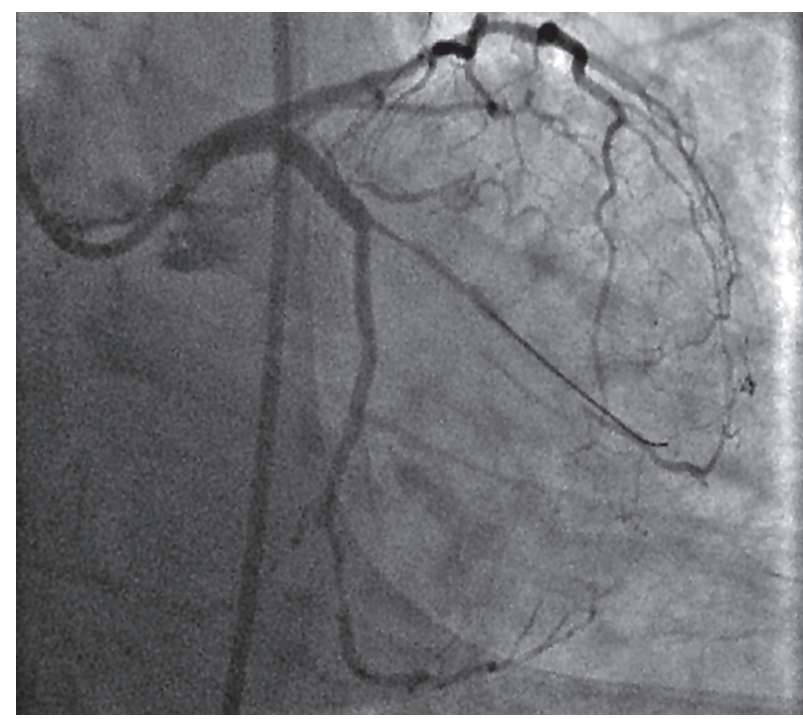

Figure 5. Wire in the distal segment

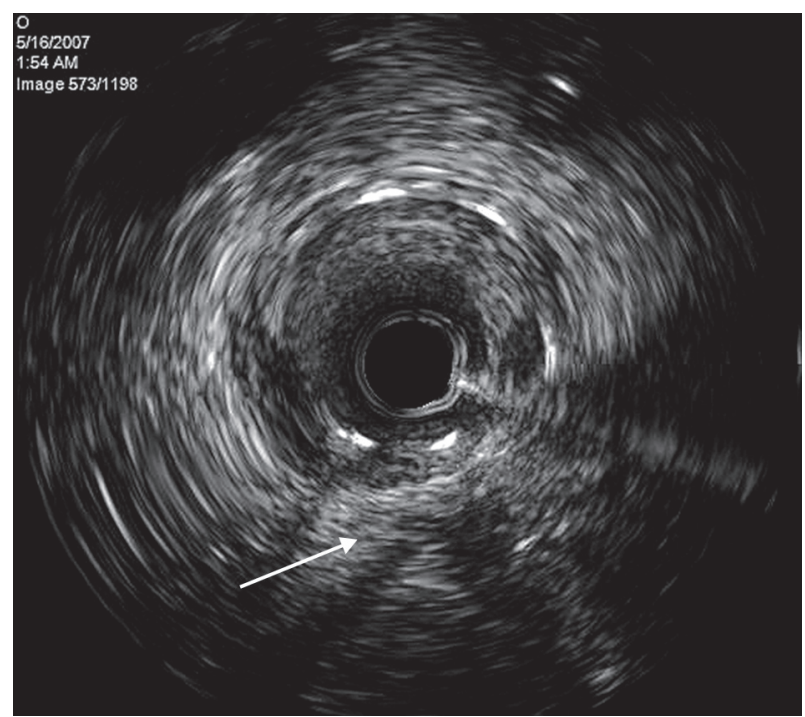

Figure 7. IVUS of the mid segment, at 3-5 o'clock position flap after the knuckled wire

\section{Discussion}

In-stent restenosis CTO is a rare complication of $\mathrm{PCl}$, with numbers around $1.7 \%$ [6]. Most patients present with stable angina. Approximately $5 \%$ of all CTO attempts are ISR CTO, with a lower success rate of $70 \%$ vs. $85 \%$ (ISR CTO vs. CTO [4]) although the data is incoherent and sparse. Failure to cross the wire or cases in which the wire slips outside and enters the subluminal space represent the most common reason to stop, and careful assessment of the wire tip from bilateral views is needed. In some cases, due to the wire going through the struts, subsequent balloon positioning is not possible. As restenotic material is mostly soft fibromuscular and fibrolipid plaque, balloon positioning should be easy. In ISR CTO, more than in other CTOs, staying in true lumen at the

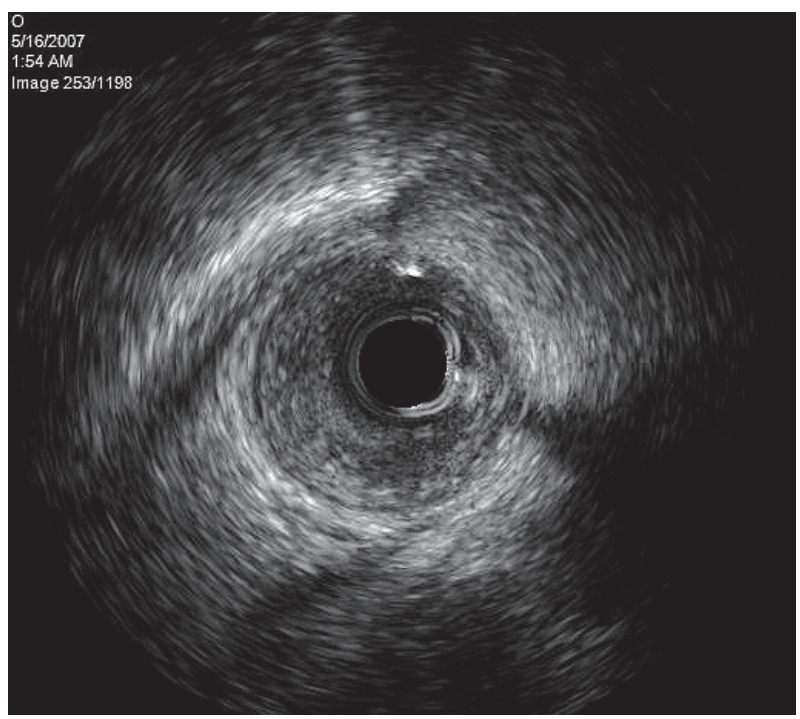

Figure 6. IVUS of the segment distal to ISR-CTO lesion

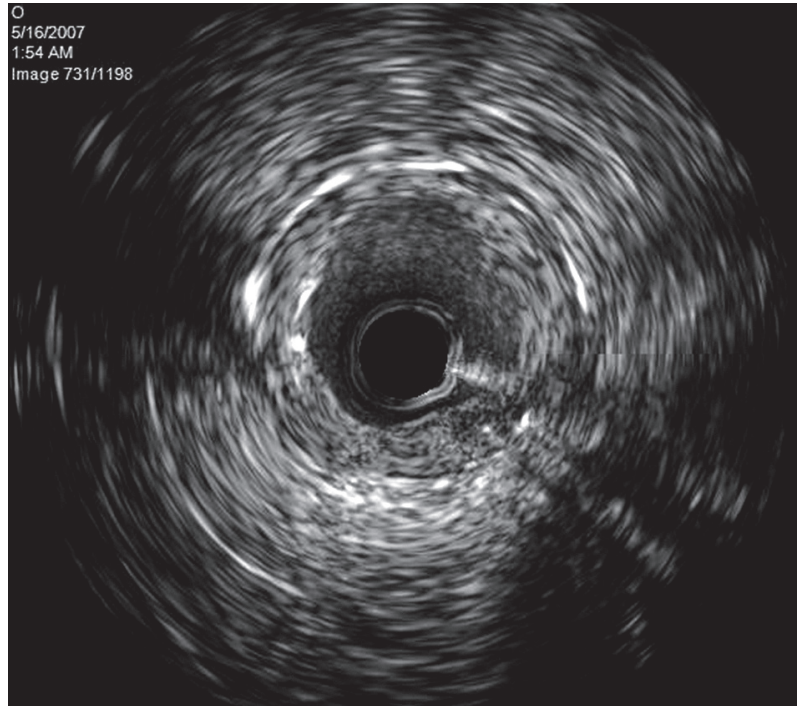

Figure 8. Most proximal part of the previously implanted stent with the mixed plaque and restenosis

point of exit of the stent is crucial. However, the distal cap of the totally occluded segment is considered easier to pass and more logical, particularly after the introduction of the retrograde technique. In our case of ISR, initial focal stenosis may have begun at that point, and penetration distally was toughest at the point of exit.

Various techniques are described to successfully cross ISR CTO, using hydrophilic soft PTFE wire as an initial wire, and a need for dedicated CTO tapered wire to push through the distal cap. Blunt dissection device was used, in some cases with success, especially since the stent is present to mark the vessel borders, but availability of the device is not widespread $[7,8]$.

In our opinion, keeping a wire inside the lumen is essential because STAR techniques in ISR CTO may lead to vessel perforation or vessel haematoma, especially when 


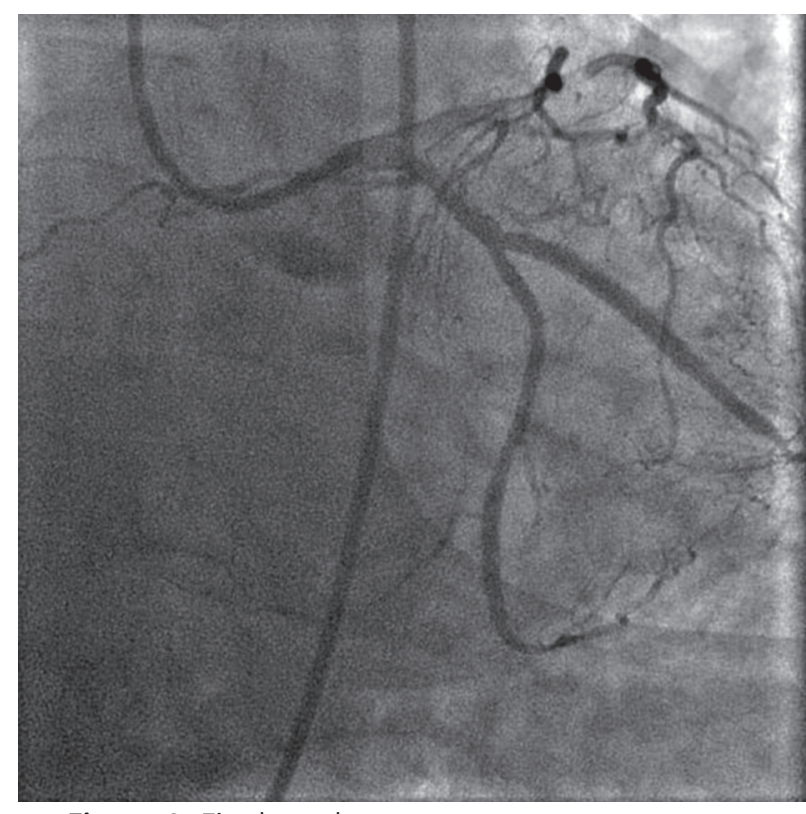

Figure 9. Final result

high dilatation pressures are needed to crush the already implanted stent. Also, newer data show high re-occlusion rates using such techniques [9]. A successful case using the STAR technique on ISR CTO was published [10].

Another problem that arises is keeping the vessel patent after the procedure as stenting for restenotic lesions, longer stent length, and smaller pre-procedural minimal luminal diameter (MLD) were independent predictors of total occlusion following coronary stenting [11]. The ISR CTO warrants excessive secondary stenting, with large plaque and neointimal load and at least two stent layers with higher inflation pressures needed to overcome stent recoil. For optimal results and optimisation additional visualisation devices should be used (IVUS, OCT).

\section{Conclusions}

We have presented a technique that does not require a dedicated device for success in crossing the ISR CTO and seems relatively safe because of specific angiographic findings in ISR CTO. Figure 10 shows the positing of the knuckle within stent struts. The roadmap provided by the previously implanted stent and the ability to check the knuckle position within the stent struts, along with the added strength of the knuckled wire, should be an option when initial crossing using usual techniques has failed.

\section{Conflict of interest}

The authors declare no conflict of interest.

\section{References}

1. Thompson CA, Jayne JE, Robb JF, et al. Retrograde techniques and the impact of operator volume on percutaneous intervention for coronary chronic total occlusions: an early U.S. experience. JACC Cardiovasc Interv 2009; 2: 834-42.

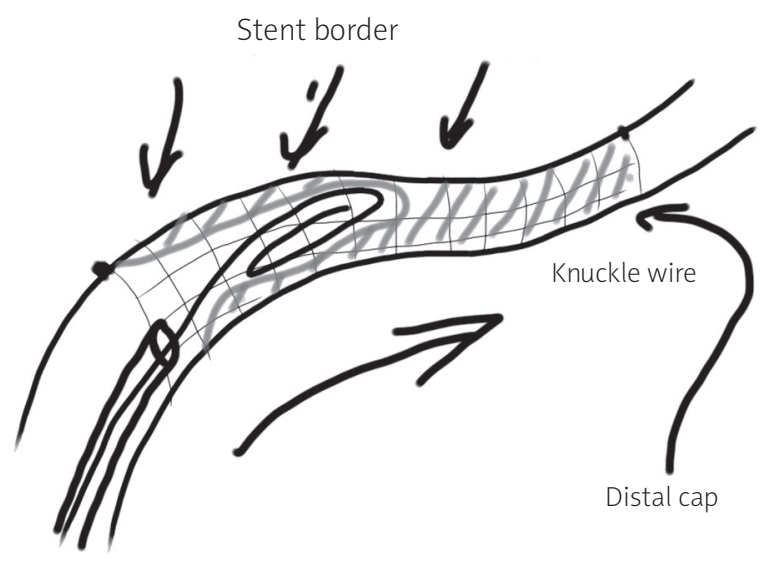

Figure 10. Schematic of the knuckle wire positioning within ISR CTO lesion

2. Farooq V, Gogas BD, Serruys PW. Restenosis: delineating the numerous causes of drug-eluting stent restenosis. Circ Cardiovasc Interv 2011; 4: 195-205.

3. Chieffo A, Foglieni C, Nodari RL. Histopathology of clinical coronary restenosis in drug-eluting versus bare metal stents. Am J Cardiol 2009; 104: 1660-7.

4. Werner GS, Moehlis H, Tischer K. Management of total restenotic occlusions. Eurolntervention 2009; 5 (Suppl D): D79-83.

5. Joyal D, Thompson CA, Grantham J, et al. The retrograde technique for recanalization of chronic total occlusions: a step-bystep approach. J Am Coll Cardiol Interv 2012; 5: 1-11.

6. Shah PB, Cutlip DE, Popma JJ, et al. Incidence and predictors of late total occlusion following coronary stenting. Catheter Cardiovasc Interv 2003; 60: 344-51.

7. Ho PC. Treatment of in-stent chronic total occlusions with blunt microdissection. J Invasive Cardiol 2005; 17: E37-9.

8. Yang YM, Mehran R, Dangas G, et al. Successful use of the frontrunner catheter in the treatment of in-stent coronary chronic total occlusions. Catheter Cardiovasc Interv 2004; 63: 462-8.

9. Valenti R, Vergara R, Migliorini A, et al. Predictors of reocclusion after successful drug-eluting stent-supported percutaneous coronary intervention of chronic total occlusion. J Am Coll Cardiol 2013; 61: 545-50.

10. Lee $\mathrm{NH}$, Cho YH, Seo HS. Successful recanalization of in-stent coronary chronic total occlusion by subintimal tracking. I Invasive Cardiol 2008; 20: E129-32.

11. Shah PB, Cutlip DE, Popma JJ, et al. Incidence and predictors of late total occlusion following coronary stenting. Catheter Cardiovasc Interv 2003; 60: 344-51. 